\title{
Imaging molecular interactions in cells by dynamic and static fluorescence anisotropy (rFLIM and EMFRET)
}

\author{
D.S. Lidke, P. Nagy, B.G. Barisas', R. Heintzmann, J.N. Post, K.A. Lidke, A.H.A. Clayton² , D.J. Arndt-Jovin \\ and T.M. Jovin ${ }^{3}$ \\ Department of Molecular Biology, Max Planck Institute for Biophysical Chemistry, 37077 Göttingen, Germany
}

\begin{abstract}
We report the implementation and exploitation of fluorescence polarization measurements, in the form of anisotropy fluorescence lifetime imaging microscopy (rFLIM) and energy migration Förster resonance energy transfer (emFRET) modalities, for wide-field, confocal laser-scanning microscopy and flow cytometry of cells. These methods permit the assessment of rotational motion, association and proximity of cellular proteins in vivo. They are particularly applicable to probes generated by fusions of visible fluorescence proteins, as exemplified by studies of the erbB receptor tyrosine kinases involved in growth-factor-mediated signal transduction.
\end{abstract}

\section{Introduction}

The anisotropy of fluorescence (or more generally, of luminescence), a measure of emission polarization, provides a very sensitive measure of molecular rotation [1]. Rotational diffusional constants scale with molecular size and thus constitute very sensitive parameters reflecting processes such as protein conformational change, dimerization, association into multimolecular structures and translocation to or integration within a membrane compartment. In the latter case, the molecule experiences a substantial viscous retardation imposed by the microenvironment, such that the resultant rotational motion is translated from the nanosecond domain characteristic of the small organic molecules free in solution to the microsecond regime. This circumstance has prompted the use of long-lived phosphorescent triplet probes instead of the conventional fluorophores [2].

The formalism required for an adequate description of emission anisotropy is simple (Figure 1). Based on dimensionless quantities (ratios of intensity functions and time constants), the measurements are relatively insensitive to technical features such as light path and absolute intensity and are therefore well suited for the microscopy of cells. Furthermore, the determination of rotational relaxation is independent of ion and oxygen concentrations, which have strong impacts on the lifetime.

Key words: erbB, Förster resonance energy transfer (FRET), fluorescence, microscopy, polarization, receptor.

Abbreviations used: FRET, Förster resonance energy transfer; emFRET, energy migration FRET; rFLIM, anisotropy fluorescence lifetime imaging microscopy; VFP, visible fluorescent protein; RTK, receptor tyrosine kinase; CLSM, confocal laser-scanning microscope; eGFP, enhanced green fluorescent protein; YFP, yellow fluorescent protein; $\mathrm{CHO}$, Chinese hamster ovary.

${ }^{1}$ Permanent address: Department of Chemistry, Colorado State University, Fort Collins, co 80525, U.S.A.

${ }^{2}$ Present address: Ludwig Institute for Cancer Research, Royal Melbourne Hospital, Parkville, Victoria 3050, Australia.

${ }^{3}$ To whom correspondence should be addressed (e-mail tjovin@gwdg.de).
Changes in emission anisotropy are also a manifestation of energy transfer between nearby or associated molecules. Thus, in the widely used technique known as FRET (Förster resonance energy transfer) [1], involving the resonance (nonradiative) transfer of energy from a photoselectively excited molecule to a nearby orientationally uncorrelated (on average) acceptor, the emission of the latter is virtually unpolarized. At the same time, the donor fluorescence is hyperpolarized (relative to the unperturbed state), due to the shortening of its excited state lifetime.

One of the most sensitive techniques for measuring timedependent fluorescent anisotropy involves the detection of the relative phase and modulation of the two polarized emission components in a frequency domain instrument. We have reported $[3,4]$ the adaptation of the concepts and experimental realization pioneered by Weber [5] and co-workers to a wide-field microscope and denoted the technique as rFLIM [anisotropy (r) Fluorescence Lifetime Imaging Microscopy].

Fluorescence depolarization occurs to the degree that the molecular frame re-adjusts during the excited state lifetime, such that the correlation between the positions in space of the absorption and emission transition dipoles diminishes from that characteristic of the immobile molecule. However, under certain conditions a second depolarization mechanism can be operative, consisting of resonance energy transfer between identical VFP (visible fluorescent protein) molecules in close proximity, a process referred to in the physics literature as energy migration RET (which we denote emFRET [3]). The magnitude of this effect can be arbitrary up to the point of complete depolarization and can be quantified by rFLIM as well as by simple static, steady-state anisotropy determinations, assuming an adequate foreknowledge of the system. We have applied both the dynamic and static techniques to studies of cellular signal transduction mediated 
Figure 1 1 Basic definitions and relations in fluorescence anisotropy

$I_{\|}$and $I_{\perp}$, respectively, are the parallel and perpendicular polarized emission components, generated by excitation with linearly polarized light. $V$ is the volume of a molecule rotating in a medium of viscosity, $\eta$. $f_{i}$ and $a_{i}$ are the fractions and amplitudes, respectively, of various anisotropy components. Other quantities appearing in the figure are defined on the figure itself or in the text.

definition

rotational relaxation

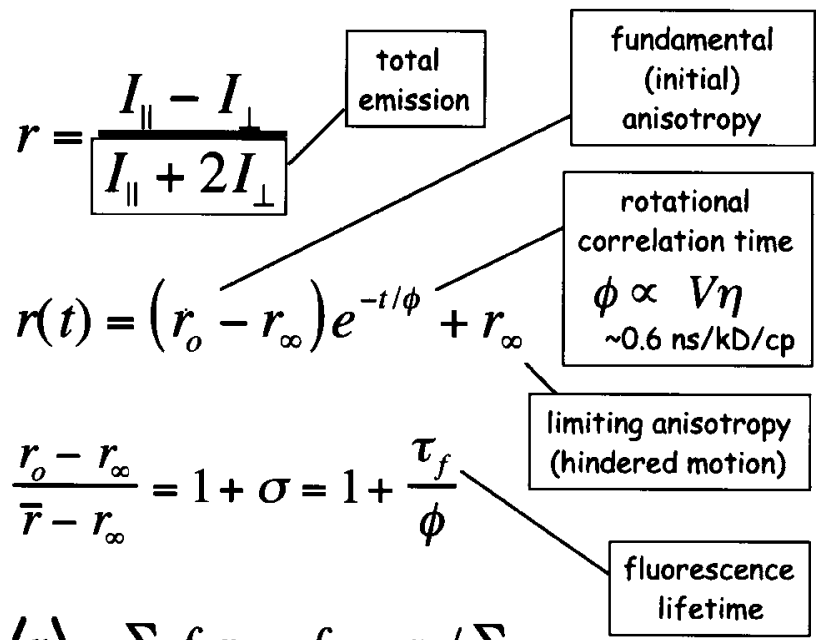

additivity rule

$\langle r\rangle=\sum f_{i} r_{i} \quad f_{i}=a_{i} / \sum a_{i}$

by RTKs (receptor tyrosine kinases) and their associated downstream cascades.

\section{rFLIM}

The microscope system initially adapted for rFLIM in our laboratory was a conventional phase-modulation frequency domain instrument incorporating the homodyne acquisition principle [3]. Linear polarizers were introduced into the excitation and emission paths and used sequentially so as to generate the required parallel and perpendicular emission images $\left(I_{\|}\right.$and $\left.I_{\perp}\right)$ recorded by an intensified CCD camera.

Three parameters are generally registered for every pixel position in the two-dimensional rFLIM images of $I_{\|}$and $I_{\perp}$ : the difference phase $(\Delta \Phi)$, the AC modulation ratio $\left(Y_{\mathrm{ac}}\right)$ and the $\mathrm{DC}$ intensity ratio $\left(Y_{\mathrm{dc}}\right)$. These quantities have a characteristic dependence on the modulation frequency of excitation (Figure 2, top row) and suffice for the determination of the three parameters of the model constituting an approximate (but generally adequate) description of the rotational diffusion of an asymmetric or hindered molecular species (Figure 1).

In applying rFLIM to molecules of interest, such as VFPs used as expression probes, we record a distinct but limited degree of rotational depolarization, in accordance with expectation [3]. Representative 'images' of an eGFP (enhanced green fluorescent protein) solution are shown in Figure 3. It is gratifying that a determination of the long rotational correlation time $(\approx 18 \mathrm{~ns})$ of this $27-\mathrm{kDa}$ protein is feasible, despite the short lifetime ( $\tau<3 \mathrm{~ns}$ ) that leads to a small value of the normalized rotational diffusion parameter, $\sigma=\tau / \Phi<0.2 ; \Phi$ is the rotational correlation time (Figures 1 and 2). Note that in this case, as well as with other molecules [3], the steady-state anisotropy $(\bar{r})$ can be used by itself as a contrast-generating parameter, i.e. without the necessity of determining $\Phi$. However, the complete three-parameter description (Figure 1) carries a much higher information content, which is particularly useful in studies of dynamic processes occurring in living cells.

The rFLIM instrument in our laboratory is currently being improved by incorporation of (i) a polarization image splitter permitting the simultaneous registration of both $I_{\|}$ and $I_{\perp}$ images by the same CCD detector, (ii) an electrooptical device for rapid modulation of the excitation polarization state, and (iii) solid-state LED and diode laser light sources. Alternative approaches for incorporating fluorescence anisotropy measurements in the microscope have been reported recently [6,7]; they differ with respect to temporal resolution (acquisition time) and means of parameter extraction.

\section{emFRET}

Conventional heterotransfer FRET arises when the emission spectrum of an excited donor chromophore overlaps that of a chemically (and spectroscopically) distinct acceptor chromophore. The phenomenon has several photophysical manifestations [1], each of which gives rise to corresponding experimental techniques for detecting the FRET efficiency: (i) sensitized emission from the acceptor chromophore (which may be but need not be fluorescent); (ii) quenching of donor fluorescence emission; (iii) shortening of donor 
Figure 2| Parametric relationships in rFLIM

Top row: dependence of the relative phase and amplitudes of the two polarized emission components as a function of the product of the radial modulation frequency given in the plots as the product with the fluorescence lifetime $\tau$, and the normalized parameter $\sigma=\tau / \Phi$ where $\Phi$ is the rotational correlation time [3]. The DC ratio (or steady-state anisotropy, $\bar{r}$, see inset) does not depend on modulation frequency. Bottom row: changes in the rFLIM parameters computed from analytical solutions of the emFRET formalism and using parameters appropriate for eGFP. The difference phase $\Delta \Phi$ increases and the AC modulation decreases as a function of the eGFP concentration $c$. The corresponding changes in $\bar{r}$ are shown with experimental data in Figure 5.
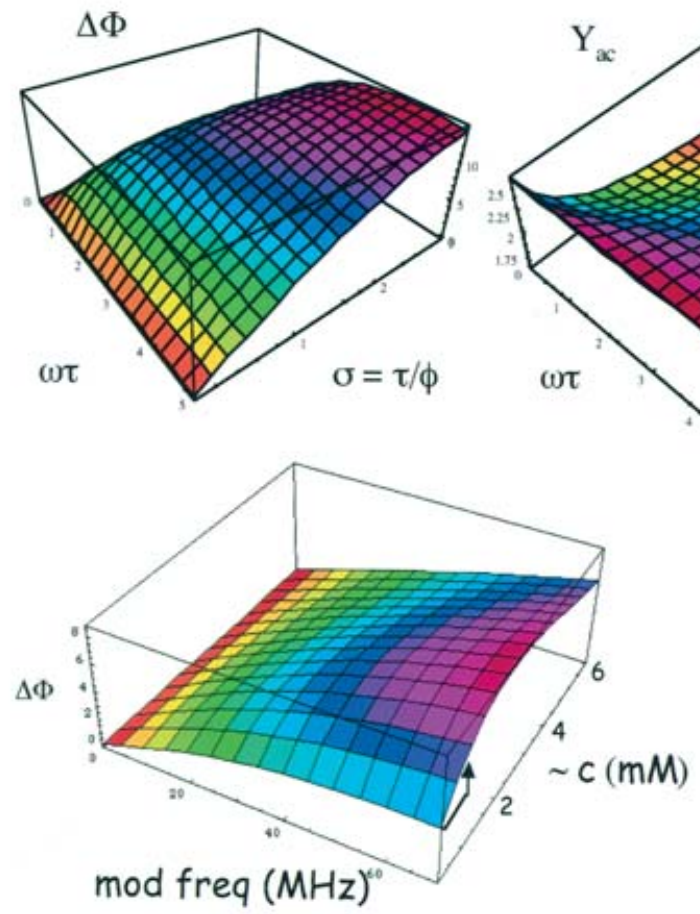
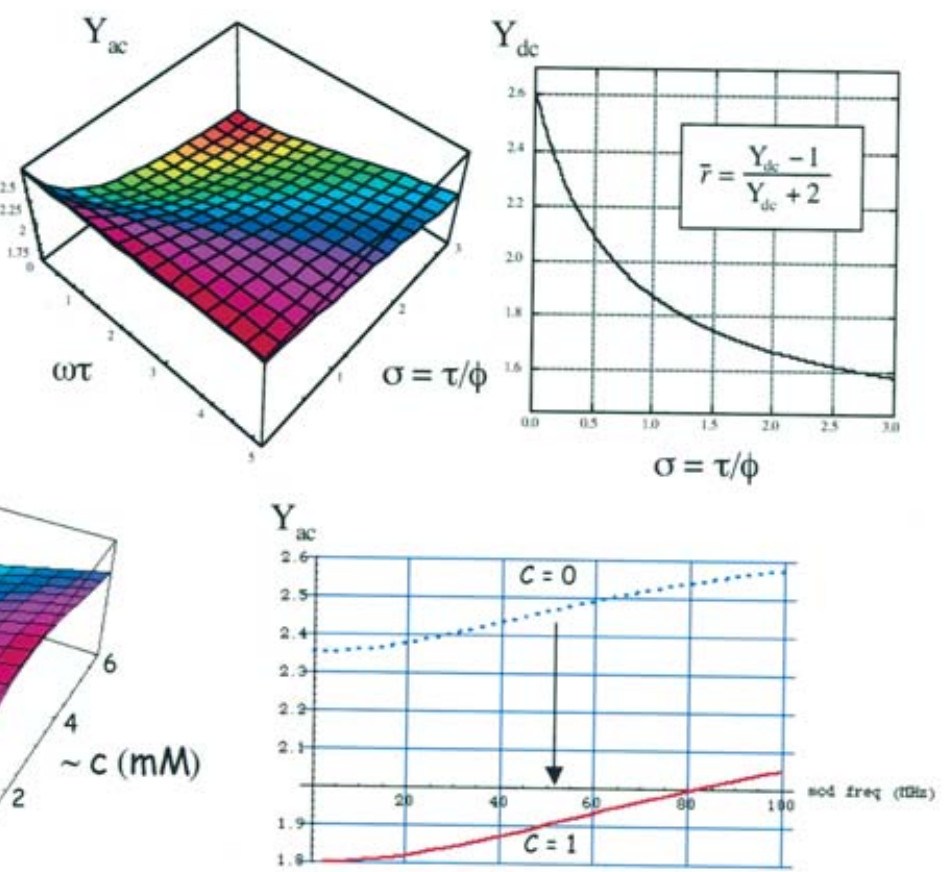

fluorescence lifetime, the basis for (ii); (iv) reduced rate of donor photobleaching $[8,9]$ and $(v)$ the polarization changes cited above.
FRET can also occur between like molecules, in the event that they exhibit a small Stokes shift, the separation between the absorption and emission spectral peaks. The Förster

\section{Figure 3 | rFLIM of eGFP in free solution}

A dilute solution of eGFP was deposited on a slide and imaged in the wide-field microscope system adapted for rFLIM measurements [3]. The measured rFLIM signals, indicated under each panel, were used to calculate the rotational diffusion parameters for an isotropic rotator $\left(r_{\infty}=0\right)$ according to [3], yielding $r_{0}=0.37$ and $\Phi=18-19$ ns.

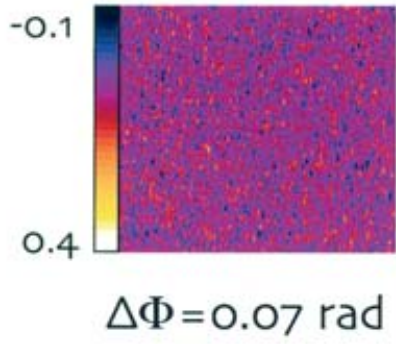

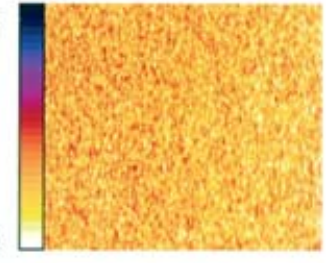

$Y_{a c}=2.56$

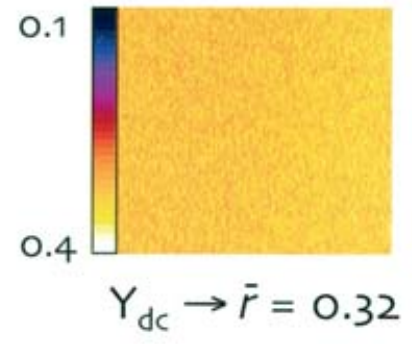




\section{Figure $4 \mid$ Principle of emFRET}

Assume a random population of like (identical) fluorophores. At low excitation intensity, molecules (one of which is designated the 'donor') will only be excited occasionally. Given a high-enough two- or three-dimensional density (concentration), excitation energy will transfer energy from the donor to a nearby 'acceptor' molecule. This process will continue, including back-transfer to the original donor, for a time dictated by the excited state lifetime. If the rate of transfer is sufficiently high, equilibration of the excitation among the ensemble of closely packed partners will occur such that on average all molecules will exhibit the same degree of fractional excitation. However, due to the randomization of orientation accompanying energy transfer, only the original donor will emit with its characteristic, intrinsic anisotropy.

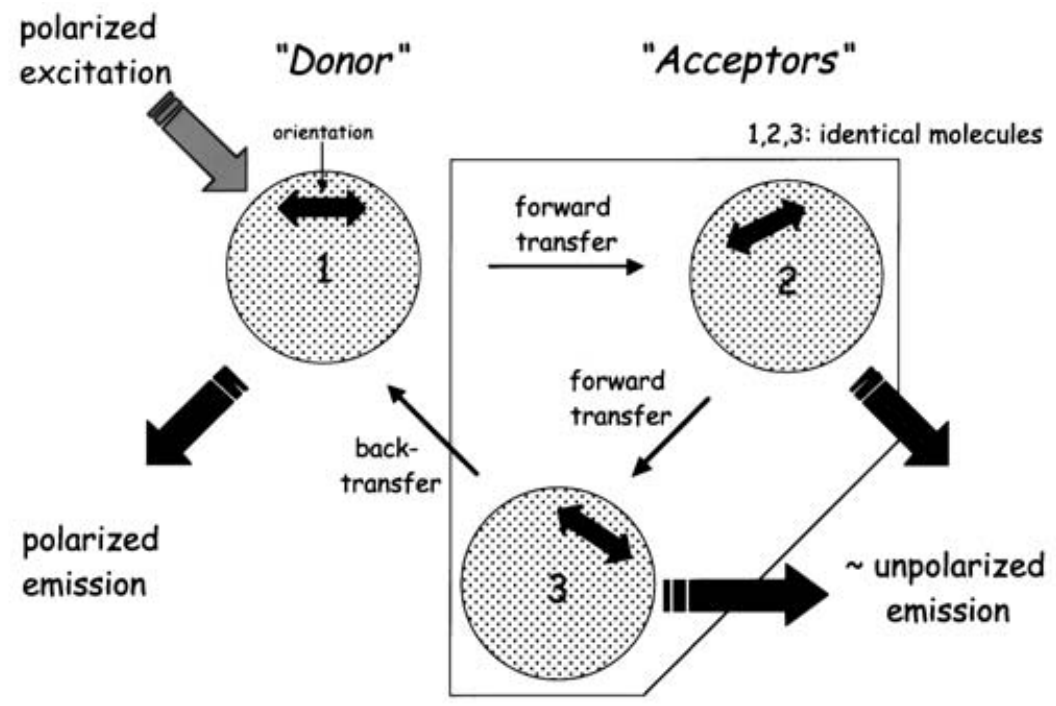

distances $\left(R_{\mathrm{o}}\right)$ for homotransfer (or emFRET) between VFP colour variants have been calculated [10], yielding rather large values, e.g. $5.1 \mathrm{~nm}$ for YFP (yellow fluorescent protein). Such quantities are comparable with those for heterotransfer FRET between common donor/acceptor pairs, for example fluorescein/rhodamine or the VFPs cyan fluorescent protein (CFP) and YFP.

In as much as emFRET occurs between like molecules, the intensity, excited state lifetime and spectral properties of the molecular ensemble remain unaltered. However, emFRET has a strong effect on the mean emission anisotropy (Figure 4). Photoselective excitation by polarized light of protein-bound dyes and VFPs with characteristically closeto-parallel absorption and emission transition moments generally leads to strongly polarized emission $[3,11]$. Since the rotation of proteins is typically slow compared with the nanosecond excited-state lifetimes of dyes, the maximal extent of the resultant depolarization is limited (Figure 1). However, if emFRET occurs, the excited molecule transfers its excitation energy to another molecule that will have a close-to-random relative orientation in the ensemble average (Figure 4). As a consequence, the anisotropy of fluorescence emitted from closely packed (as in concentrated solutions) and/or associated molecules undergoing emFRET is substantially reduced in comparison with that exhibited in the isolated state. For example, in the limit of very efficient transfer between randomly orientated chromophores, the steadystate anisotropy of an oligomer constituted of $n$ monomers will be $n^{-1}$ that of the monomer [12]. A very important practical virtue of emFRET in live cell studies is the requirement for only a single expression probe, obviating the necessity for the balanced expression of two distinct probes in the same cell, which is often difficult to achieve.

In the rFLIM technique, emFRET leads to a characteristic increase in $\Delta \Phi$ and a decrease in the AC modulation and DC intensity ratios ( $Y_{\mathrm{ac}}$ and $Y_{\mathrm{dc}}$; Figure 2, bottom row). However, for many applications it suffices to implement the determination of emFRET by steady-state anisotropy. We have accomplished this aim in the wide-field microscope, the confocal laser-scanning microscope (CLSM), and the flow cytometer. These instruments yield complementary and mutually confirmatory information. In the remainder of this article we explore briefly each of these approaches and evaluate their relative strengths and challenges.

\section{Static fluorescence anisotropies of molecules in solution}

emFRET between pairs of a given molecular species in free solution or a cellular compartment such as the plasma membrane leads to a reduction of $\bar{r}$ in the process of concentration depolarization (as discussed above). The appearance of emFRET to a degree that exceeds that predicted from the bulk concentration or local density alone is indicative of self-association. Thus, the concentration dependence of emFRET can provide a measure of molecular self-association 
Figure 5 | Concentration depolarization (emFRET) of the VFP Venus (a) and its A206K mutant (b)

Plotted are $\bar{r}$ and the total emission intensity corrected for the inner-filter effect on excitation in a $0.25 \mathrm{~mm} \times 0.25 \mathrm{~mm}$ microcuvette. Excitation, $520 \mathrm{~nm}$; $\bar{r}$ calculated by a weighted average over the emission range 520-550 nm.
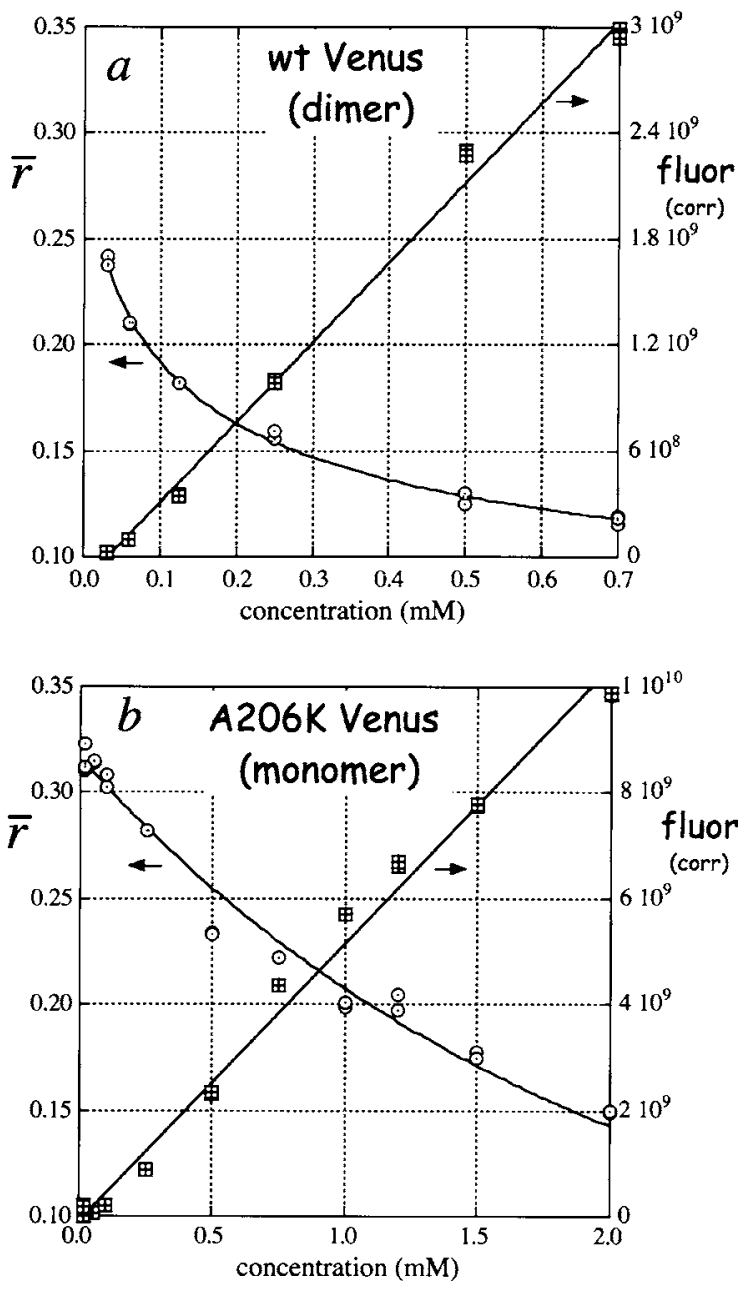

if the corresponding dissociation constants are low enough. Various lines of evidence suggest that VFPs exhibit this characteristic, albeit to a varying degree.

We have expressed His-tagged VFPs in Escherichia coli and measured the anisotropies of the purified proteins as a function of concentration in a Varian (Cary) Eclipse spectrofluorometer. To minimize inner-filter effects, a microcuvette with a $0.25 \mathrm{~mm} \times 0.25 \mathrm{~mm}$ cross-section was used. Figure 5(a) depicts the fluorescence anisotropy concentration dependence for the VFP denoted Venus, whose spectral properties closely resemble that of citrine and YFP [13]. The emission anisotropy decreases sharply with increasing protein concentration, whereas the fluorescence intensity (straight line), corrected for inner-filter effects, increases linearly, confirming a lack of quenching. The question is whether simple concentration depolarization is operative in this system. Substantial energy transfer would be expected at concentrations on the order of 1 molecule/sphere of radius $R_{\text {o }}$, i.e. $\approx 1 \mathrm{mM}$; see [3] for a detailed treatment. Thus, the strong curvature of the anisotropy plot in Figure 5(a), already evident at $0.1 \mathrm{mM}$, is indicative of a monomer-dimer equilibrium, resulting from strong emFRET between the monomer units comprising the dimer. A quantitative analysis of the data yields a dissociation constant of $0.19 \pm 0.04 \mathrm{mM}$ (Figure 5a).

Other VFPs exhibit a greater or lesser tendency for selfassociation. In the event that they remain or can be rendered monomeric, which can be generally achieved by a strategic A206K mutation [14], emFRET is restricted to the concentration depolarization regime corresponding to higher concentrations. In the case of the A206K mutant of Venus, this is indeed observed (Figure $5 \mathrm{~b}$ ) and analysis yields an $R_{\mathrm{o}}$ of $5.9 \mathrm{~nm}$. The apparent value for eGFP is even higher $(7.3 \mathrm{~nm}$ [3]), a result implying the need for further exploration of the formalism used to quantify the concentration depolarization of VFPs. The marked differences between the dimerizing (wild-type) and monomerized Venus, and other VFPs are reflected in the properties of their respective fusion proteins with receptor tyrosine kinases (see below), leading to the conclusion that the inherent tendency of most VFPs to self-associate may have profound, generally undesirable, structural and thus functional consequences in the cellular context. It is fortunate that the eGFP widely used in cell biological studies does not dimerize to an appreciable extent [3].

\section{CLSM of cell-surface proteins displaying emFRET}

To the extent that dyes and VFP fusion proteins photobleach during continuous or sequential observation, the efficiency of emFRET will decrease in a systematic manner as bleaching progresses, reflecting the reduction in fluorophore density. (Note that in the case of VFPs, the protein 'shells' and thus molecular complexes will remain largely unaltered after photobleaching). This dependency is reflected in and monitored quantitatively via the emission anisotropy. Thus, an increase in this quantity from a low initial value as a function of the extent of photobleaching constitutes strong evidence for emFRET and, consequently, for close molecular association and/or high local density of the molecule(s) in question.

We have employed quantitative microscopy in numerous studies of RTKs, transmembrane proteins mediating signal transduction in response to external regulatory ligands such as growth factors [15]. A central dogma has been the notion of dimerization of such proteins as a consequence of ligand binding to the ectodomains and as a prerequisite for activation of the latent protein kinase in the cytoplasmic domain [16]. We reported previously evidence based on FRET-FLIM imaging of the epidermal growth factor receptor (erbB1), suggesting that a substantial fraction of such receptors might be preassociated (e.g. dimerized) in the absence of ligand [17]. In view of supporting evidence accumulating from other sources, these experiments have been extended to wide-field 
Figure 6 | Anisotropy image of a CHO cell expressing erbB1-eGFP and activated by epidermal growth factor

Cells were exposed to epidermal growth factor (EGF) for $5 \mathrm{~min}$ at $4^{\circ} \mathrm{C}$, incubated for 5 min at $37^{\circ} \mathrm{C}$, and fixed in methanol. Left panel: intensity image, the brighter regions indicating receptor aggregation in response to activation by EGF. Central panel: grouping of pixel intensities into colour-coded low, medium and high classes. Right panel: anisotropy image calculated with the $I_{\|}$and $I_{\perp}$ images taken in the CLSM. The regions of medium and high intensities, in which the protein was at higher concentration, show lower anisotropy values.

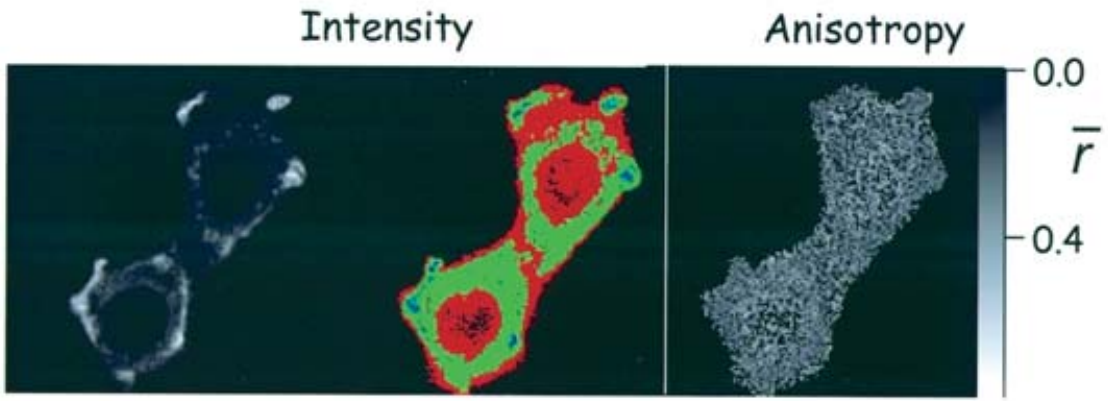

red: low; green: middle; blue: high

microscopy and CLSM of VFP fusions with members of the erbB RTK family, exploiting emFRET of the VFPs with the dynamic and static anisotropy techniques. The homologous and heterologous association of various erbB molecules expressed on tissue-culture cell lines was assessed. Anisotropy measurements were performed using a Zeiss model 310 CLSM adapted with a polarizing beam splitter directing the two polarized emission components to dual photomultipliers for simultaneous detection. To reduce photophysical saturation of the fluorophores during imaging, image collection at a $10 x$ attenuated laser power alternated with sample bleaching with the unattenuated laser beam. Image sequences were corrected for background intensities from a cell-free region of the same frame and the anisotropies calculated on a pixel-by-pixel basis.

Various methods of image analysis can be applied to such data, e.g. the sorting of pixels into three categories of signal intensity (low, medium, high; Figure 6). Higher concentrations were generally correlated with lower anisotropy, indicative of concentration depolarization (emFRET), as shown in Figure 6 for the erbB1-eGFP construct stably expressed at high levels in $\mathrm{CHO}$ (Chinese hamster ovary) and A431 human epidermoid carcinoma cell lines. One way to proceed with such data is to compute the mean anisotropies of the three intensity classes during the course of cellular manipulations. We have obtained trend lines characteristic for different states of the RTKs during activation and internalization stages of signal transduction using this procedure.

Alternatively, the data can be averaged for an entire cell, as shown in Figure 7 for the same erbB1-eGFP-expressing lines. The anisotropy of the fluorescent receptor on $\mathrm{CHO}$ cells increased substantially as the sample photobleached during sequential scans, whereas the values for the A431 cells remained relatively constant. Since $\mathrm{CHO}$ cells do not express intrinsic erbB1, a tendency to self-association of erbB1-
eGFP expressed in the transfectant would result in the formation of homodimers (or higher homo-oligomers) with emFRET between the constituent eGFPs. In contrast, erbB1 self-association on A341 cells, which express high levels $\left(\approx 2 \times 10^{6}\right.$ [18]) of endogeneous (non-fluorescent) erbB1, would presumably involve a substantial fraction of dimers consisting of a non-fluorescent and an eGFP-labelled erbB1 receptor. Such species would not exhibit significant emFRET, a result in agreement with the observation of relatively high initial $\bar{r}$ values, which did not increase upon photobleaching. For the CHO transfectants, pixel-by-pixel analysis indicated that the highest intensity pixels, i.e. of highest local receptor concentrations, exhibited the lowest anisotropies, reflecting a higher extent of association in these regions. This figure

Figure 7 | Mean cellular anisotropy of erbB1-eGFP expressed in CHO and A431 cells as a function of fractional residual fluorescence See text for discussion.

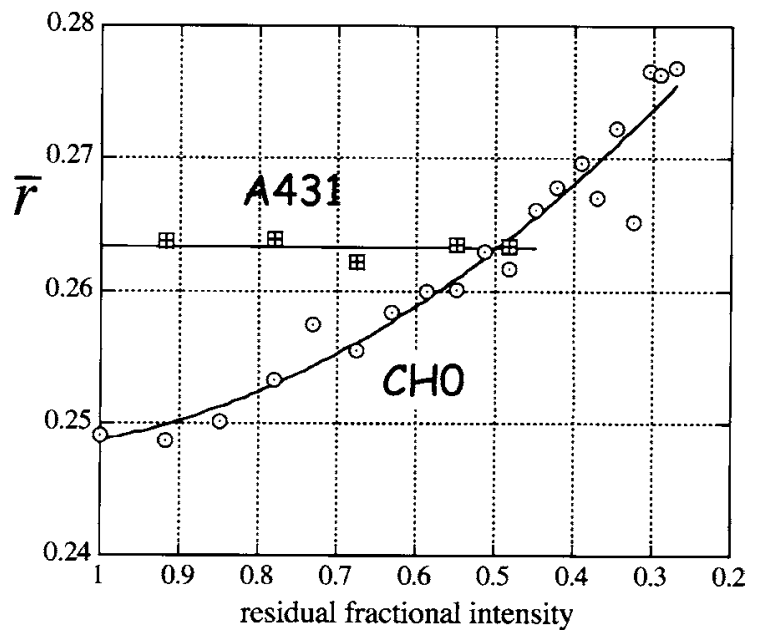


Figure 8 | Anisotropy distributions determined by flow cytometry of CHO and A431 cells expressing erbB1-eGFPn The anisotropy of $20000 \mathrm{CHO}$ (a) and A431 (b) cells expressing erbB1-GFP was determined by flow cytometry. Single-cell anisotropy values (black dots) have been plotted as a function of total $\left(I_{\|}+2 I_{\perp}\right)$ eGFP fluorescence intensity. The mean anisotropy corresponding to cells with a given total eGFP fluorescence is displayed as the trend line.
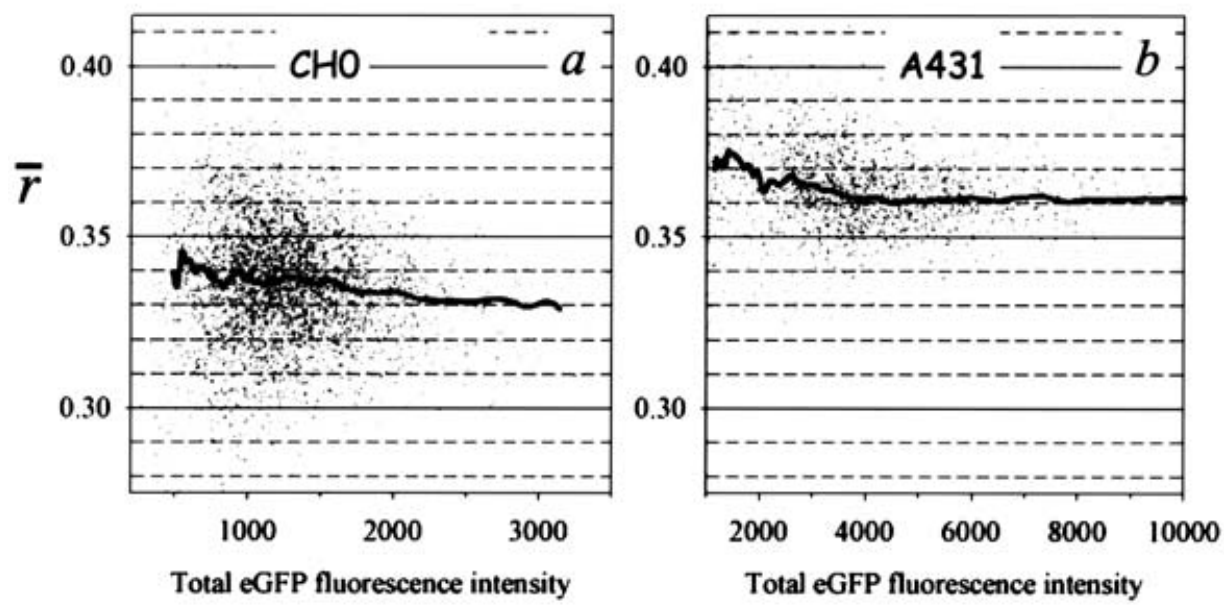

also illustrates the advantage of the emFRET method alluded to above, i.e. that only a single molecular species needs to be transfected into a cell to evaluate intermolecular association. However, a careful and quantitative correction for background contributions to the images is essential for accurate anisotropy determinations with cells.

\section{emFRET measurements by polarization flow cytometry}

The flow cytometer offers a valuable adjunct to the microscope because it generates very accurate cellular population distributions, albeit with the loss of subcellular resolution due to the acquisition of signals derived from whole cells. A detailed description of polarization detection in flow cytometers has been presented elsewhere [19]. We have adapted a Coulter Epics Elite flow cytometer for simultaneous detection of the two polarized emission components.

Despite the lack of subcellular resolution, the flow cytometer provides a reliable measurement of the association of erbB1-eGFP RTKs, since $\approx 90 \%$ of the expressed protein is located in the plasma membrane of stably transfected cells. Since cells traverse the focused laser source only once, it is not possible to carry out the photobleaching-resolved emFRET measurements described above. However, one can correlate cell-by-cell $\bar{r}$ values with whole cell expression levels by recourse to the multiparametric acquisition and recording capabilities inherent to flow cytometric technology.

$\mathrm{CHO}$ cells lack erbB1 whereas A431 cells overexpress this RTK to a level of $2 \times 10^{6}$ erbB1 molecules/cell. Both cell lines were transfected with erbB1-eGFP. CHO and A431 cells with erbB1-eGFP showed mean $\bar{r}$ values of $\approx 0.34$ and $\approx 0.36$, respectively (Figure 8 ), indicating a somewhat higher degree of erbB1-GFP homoassociation in CHO cells, in accordance with the findings of the imaging experiments discussed above. In addition, the anisotropy of erbB1-eGFP in $\mathrm{CHO}$ cells displayed a monotonic decrease as a function of GFP fluorescence intensity, whereas the trend in A431 cells was more biphasic. This relative concentration dependence, in this case derived from the natural distribution of expression in the cell population instead of photobleaching, was also indicative of preformed erbB1 complexes. Finally, we note that the mean anisotropy of erbB1-eGFP in the cell was lower than the $r_{\mathrm{o}}$ of free eGFP $(\approx 0.39$, Figure $1[3])$.

\section{Concluding remarks}

The complexity of living cells necessitates an interdisciplinary experimental strategy for their study. In this report, we have featured the virtues of fluorescence anisotropy as a means for assessing the structural and functional states of biological molecules. Anisotropy-based methods offer a number of advantages, particularly for the study of homotropic interactions of and between biomolecules. Such interactions, represented widely in the biochemical networks of the cell, can be evaluated by emFRET upon introduction and expression of only a single protein. These proteins can then be tagged intrinsically with a VFP or externally with rigid biarsenical dyes, i.e. FlAsH [20]. Another virtue of these methods is the ability to image anisotropy both statically and dynamically, allowing characterization of intermolecular interactions within specific cellular compartments and with high temporal and spatial resolution. rFLIM and emFRET are compatible with other related biophysical methodologies, such as 
measures of translational diffusion and reaction (based on photobleaching, photoactivation, and/or temporal and spatial correlation), total internal reflection microcopy, single molecule detection and scanning probe microscopy.

D.S.L. and R.H. are supported by European Union FP 5 Project QLG2-2CT-2001-02278 and P.N. from Project QLG1-CT-2000-01260. B.G.B. is a recipient of a Humboldt Research Award from the Alexander von Humboldt Foundation, of NSF grant DBI-0138322, and of a grant from the Colorado State University CVMBS Council. J.N.P. is supported by grant AR 246-2 to D.J.A.-J. from the SPP Program 1050 of the Deutsche Forschungsgemeinschaft. A.H.A.C. was a recipient of a long-term postdoctoral fellowship from the Human Frontier Science Program.

\section{References}

1 Valeur, B. (2002) Molecular Fluorescence: Principles and Applications, Wiley-VCH, Weinheim

2 Jovin, T.M. and Vaz, W.L. (1989) Methods Enzymol. 172, 471-513

3 Clayton, A.H.A., Hanley, Q.S., Arndt-Jovin, D.J., Subramaniam, V.S. and Jovin, T.M. (2002) Biophys. J. 83, 1631-1649

4 Subramaniam, V., Hanley, Q.S., Clayton, A.H.A. and Jovin, T.M. (2003) Methods Enzymol. 360, 178-201
5 Weber, G. (1977) J. Chem. Phys. 66, 4081-4091

6 Gautier, I., Tramier, M., Durieux, C., Coppey, J., Pansu, R.B., Nicolas, J.C., Kemnitz, K. and Coppey-Moisan, M. (2001) Biophys. J. 80, 3000-3008

7 Siegel, J., Suhling, K., Leveque-Fort, S., Webb, S.E.D., Davis, D.M., Phillips, D., Sabharwal, Y. and French, P.M.W. (2003) Rev. Sci. Instr. 74, 182-192

8 Jovin, T.M. and Arndt-Jovin, D.J. (1989) in Cell Structure and Function by Microspectrofluorometry (Kohen, E. and Hirschberg, J.G., eds.), pp. 99-117, Academic Press, San Diego, CA

9 Young, R.M., Arnette, J.K., Roess, D.A. and Barisas, B.G. (1994) Biophys. J. 67, $881-888$

10 Patterson, G.H., Piston, D.W. and Barisas, B.G. (2000) Anal. Biochem. 284, 438-440

11 Volkmer, A., Subramaniam, V., Birch, D.J. and Jovin, T.M. (2000) Biophys. J. 78, 1589-1598

12 Runnels, L.W. and Scarlata, S.F. (1995) Biophys. J. 69, 1569-1583

13 Nagai, T., Ibata, K., Park, E.S., Kubota, M., Mikoshiba, K. and Miyawaki, A. (2002) Nat. Biotechnol. 20, 87-90

14 Zacharias, D.A., Violin, J.D., Newton, A.C. and Tsien, R.Y. (2002) Science 296, 913-916

15 Yarden, Y. and Sliwkowski, M.X. (2001) Nat. Rev. Mol. Cell Biol. 2 127-137

16 Schlessinger, I. (2002) Cell 110, 669-672

17 Gadella, Jr, T.W.J. and Jovin, T.M. (1995) J. Cell Biol. 129, 1543-1558

18 Nagy, P., Arndt-Jovin, D. and Jovin, T.M. (2003) Exp. Cell Res. 285, 39-49

19 Bene, L., Fulwyler, M.J. and Damjanovich, S. (2000) Cytometry 40, 292-306

20 Zhang, J., Campbell, R.E., Ting, A.E. and Tsien, R.Y. (2002) Nat. Rev. Mol. Cell Biol. 3, 906-918

Received 7 July 2003 